\title{
Visual and radiographic caries detection: a tailored meta-analysis for two different settings, Egypt and Germany
}

\author{
Falk Schwendicke ${ }^{1 *}$, Karim Elhennawy ${ }^{1}$, Osama El Shahawy ${ }^{2,3}$, Reham Maher $^{2,3}$, Thais Gimenez ${ }^{4}$, \\ Fausto M. Mendes ${ }^{4}$ and Brian H. Willis ${ }^{5}$
}

\begin{abstract}
Background: Diagnostic meta-analyses on caries detection methods should assist practitioners in their daily practice. However, conventional meta-analysis estimates may be inapplicable due to differences in test conduct, applied thresholds and assessed population between settings. Our aim was to demonstrate the impact of tailored meta-analysis of visual and radiographic caries detection to different settings using setting-specific routine data.

Methods: Published systematic reviews and meta-analyses on the accuracy of visual and radiographic caries detection were used. In two settings (a private practice in Germany and a public health clinic in Egypt), routine data of a total of 100 ( $n=50 /$ practice) consecutive 12-14 year-olds were collected. Test-positive rates of visual and radiographic detection for initial and advanced carious lesions on occlusal or proximal surfaces of molars were used to tailor meta-analyses. If prevalence data were available, these were also used for tailoring.

Results: From the original reviews, 210 and 100 heterogeneous studies on visual and radiographic caries detection were included in our meta-analyses. For radiographic detection, sensitivity and specificity estimates derived from conventional and tailored meta-analysis were similar. For visual detection of advanced occlusal carious lesions, the conventional meta-analysis yielded a sensitivity and specificity (95\% Cl) of 64.6\% (57-71) and 90.9\% (88-93), whereas the tailored estimates for Egypt were $75.1 \%$ (70-81) and 84.9\% (82-89), respectively, and $43.7 \%$ (37-51) and 96.5\% (95-97) for Germany, respectively.

Conclusion: Conventional test accuracy meta-analyses may yield aggregate estimates which are inapplicable to specific settings. Routine data may be used to produce a meta-analysis estimate which is tailored to the setting and thereby improving its applicability.
\end{abstract}

Keywords: Caries detection, Decision making, Diagnostic accuracy studies, Evidence-based dentistry, Medical informatics

\section{Background}

To detect carious lesions, dentists can use a number of methods, namely visual or visual-tactile detection, radiography, or further methods employing, for example, laser-fluorescence or near-infrared light. The accuracy of the methods is measured in terms of their sensitivity and specificity. The more sensitive methods allow the detection of early lesions, which facilitates non- or

\footnotetext{
* Correspondence: falk.schwendicke@charite.de

${ }^{1}$ Department of Operative and Preventive Dentistry, Charité -

Universitätsmedizin Berlin, Aßmannshauser Str. 4-6, 14197 Berlin, Germany

Full list of author information is available at the end of the article
}

micro-invasive treatment $[1,2]$, but they can also lead to over-detection and overtreatment. The risks of over- and under-detection further depend on the chosen cut-off for a positive result and how the test is conducted [3-6] - does one practitioner look or search more intently for lesions than another for instance?

Theoretically, the sensitivity and specificity, and hence risks of over- and under-detection, may be determined by a Diagnostic Accuracy Study (DAS). However, DAS may vary significantly in how visual or radiographic assessment is applied, the threshold for a positive result and the type of target disorder being investigated. The

(c) The Author(s). 2018 Open Access This article is distributed under the terms of the Creative Commons Attribution 4.0 International License (http://creativecommons.org/licenses/by/4.0/), which permits unrestricted use, distribution, and reproduction in any medium, provided you give appropriate credit to the original author(s) and the source, provide a link to the Creative Commons license, and indicate if changes were made. The Creative Commons Public Domain Dedication waiver (http://creativecommons.org/publicdomain/zero/1.0/) applies to the data made available in this article, unless otherwise stated. 
test positive rate (the proportion of those who tested positive in all those tested) captures some of these features.

Moreover, DAS are often performed on different populations (many DAS on caries are performed in vitro), with different prevalence rates and patient case-mixes. This is well recognized and is sometimes described as 'spectrum bias' or more accurately 'spectrum effects' and is known to affect test performances [7]. Diagnostic meta-analysis aims to accommodate this heterogeneity [8], and provides estimates for the average sensitivity, specificity and positive or negative likelihood ratios $[3,6]$.

However, an average estimate may be unrepresentative of an individual dental practitioner who may use a different threshold, execute the test differently and see a population of patients which is quite different from that represented by the average sensitivity and specificity $[3,6,9]$.

In response to this challenge, tailored meta-analysis (TMA) has been developed [10, 11]. Essentially the method combines routine data collected from the setting with the research data from DAS. In particular, routine data can often be collected to estimate the test positive rate and, in some cases, the prevalence. The novel aspect of the method lies in the exploitation of logical relations between the test positive rate, prevalence, sensitivity and specificity. Thus, if the first two are known it allows us to deduce the ranges of values for the sensitivity and specificity, and these may be used to tailor the selection of studies to the setting of interest for meta-analysis. Importantly, when the sensitivity and specificity for a detection method is tailored to the setting of interest this affects the positive and negative predictive values for the test in the setting, which may ultimately affect treatment decisions.

The present study used TMA to estimate the accuracy of visual and radiographic caries detection in two very different settings, Germany and Egypt. As an example, we used a population of 12- to 14-year olds, with caries detection on proximal and occlusal surfaces of permanent molars. We aimed at providing specific accuracy estimates for the two settings, hypothesizing that tailored accuracies differ between settings and from those yielded by conventional meta-analysis.

\section{Methods}

\section{Study design}

Conventional diagnostic meta-analyses synthesize all available data on a specific detection method. In contrast, TMA synthesizes only those data applying to a specific setting and/or particular examiners, for example with regards to test positive rate or prevalence. The present study performed TMA using three different data sources: (1) Two published systematic reviews and meta-analyses of DASs on visual and radiographic caries detection; (2) Routine data from a private practice in Germany and a public health clinic in Egypt were collected to estimate the respective test-positive rates; (3) Prevalence data for visually detected occlusal carious lesions were collected for both settings.

These are now discussed below.

\section{Systematic reviews}

Details on the systematic review processes have been described elsewhere $[3,6]$. Briefly, two reviewers searched PubMed, Embase and Cochrane Central for studies reporting accuracy data on visual [3] or radiographic detection of carious lesions [6]. The included studies reported a reference test (often histological assessment, but also other reference standards like invasive opening of the presumed lesion) to measure the performance of the index test (visual or radiographic detection). Both in vitro (the majority of studies) and clinical studies were included.

For our study, only those studies which reported sufficient data to estimate the sensitivity and specificity of the method for the proximal and/or occlusal surfaces of permanent teeth were considered. We extracted true positive, true negative, false positive and false negative numbers for each study. Data were separately extracted for studies on

1. radiographic or visual caries detection

2. detection of occlusal or proximal surfaces

3. initial and advanced lesions or only advanced lesions

Note that the definition of initial and advanced lesions was not standardized; most studies regarded cavitated lesions or those clinically and/or radiographically extended in dentin as advanced, which left non-cavitated lesions or those confined to enamel as being initial. Such dichotomization is useful, as the threshold of cavitation or dentin involvement is often used to decide between non-invasive (non-operative) and invasive (operative, restorative) treatment.

Overall, eight different subsets of data were extracted (data on visual caries detection on proximal surfaces were later discarded, as accessibility of these surfaces varied widely). As not all of the studies reported on each subset, the number of included studies varied considerably between subsets. The data used for each subset may be found in the Additional file 1 .

\section{Routine data collection}

For the present study, routine data were defined as data routinely collected on a population of patients without specific scientific purpose. Routine data thus carry the advantage that they will be available from a large range 
of settings, (for example claims data) and capture real-life practice. Routine data collection was approved by the local ethics committees (Charité ethics EA2 137/ 14; Cairo university ethics 16/7/3), and only anonymized aggregated data were used for statistical evaluation.

Over 6 months, routine examination data were collected on 50 consecutive patients aged 12-14 years old attending for a regular dental examination in each of the settings. This age was chosen, as the data were available to estimate the test positive rate and prevalence for 12-year olds in both Germany and Egypt. Patients not falling into this age band or where their data were not fully available (for example due to non-compliance with the diagnostic process) were excluded.

The data were collected by two examiners, one in each setting, and each having more than 15 years of experience in dental practice as well as experience in visual and radiographic lesion detection and evaluation.

The private practice in Germany served approximately 3000 regularly attending patients and was situated in a mid-sized town in rural Northern Germany. Regular epidemiologic surveys indicate comparatively high caries experience in this area $[12,13]$. The university clinic in Egypt served approximately 250 patients per day and was situated in Giza, metropolitan region of Cairo.

Visual caries detection on molars was performed using ICDAS criteria [14], with ICDAS scores 1 and 2 being recorded as "initial lesions" and scores $3-6$ as "advanced lesions". Tooth cleaning was not routinely performed prior to the assessment, but calculus and plaque were removed if considered to be needed by the dentist. A standard dental mirror (of different manufacturers both within and between practices), a straight-ended dental probe and a 3-in-1 air/water syringe in standard dental chairs (Germany: Dentorest, Ritter, Biberach, Germany; Egypt: Belmont clesta, Takara Belmont, Tokyo, Japan) were used, with teeth being dried during examination. Cotton rolls were not regularly used during examination.

Data on visual detection were recorded only for occlusal surfaces, as visual accessibility of proximal surfaces in this age group varied greatly between patients due to different eruption status of teeth. For sealed teeth, detection was performed as far as the sealant allowed; in most cases, the surface was deemed not assessable. Restored occlusal surfaces were excluded as well. If more than one lesion was detected per occlusal surface, the worst score was recorded.

Radiographic bitewing caries detection was performed for both occlusal and proximal surfaces. In Germany, analogue Kodak Insight films (Eastman Kodak, Rochester, NY, USA) were used. Films were exposed for $0.08 \mathrm{~s}$ at $70 \mathrm{kVp}$ and $7 \mathrm{~mA}$, and developed in a Dürr Dental XR 24 processing machine (Dürr, Bietigheim-Bissingen, Germany). In Egypt, Kodak Intraoral E-speed films were exposed for $0.12 \mathrm{~s}$ at $70 \mathrm{kVp}$ and $7 \mathrm{~mA}$, and manually developed. Films were used in various bitewing holders (this varied across but also within settings, given this being routine application). A scoring system describing lesions in outer and inner enamel (E1, E2) and outer, middle and inner dentin (D1-3) was used. Surfaces not assessable (due to radiographic projection, or restorations or orthodontic bands present) were excluded, as were third molars. Scores were rescored into initial (E1, E2) and advanced lesions (D1-D3). Data on detection of non-occlusal and non-proximal detection were not included.

Thus, for each of the tests (visual inspection or radiography) routine data from each of the two settings were used to describe the number of lesions (initial or advanced) as a proportion of the total number of assessed (occlusal or proximal) surfaces. This enabled an interval estimate for each of the respective test positive rates.

\section{Estimation of the prevalence}

For the two settings, we estimated the prevalence rates of visually detected initial and advanced, as well as only advanced occlusal carious lesions. For Germany, data from a city of similar size location and socioeconomic structure and a similar population of rather high risk children were used [15]. We assumed $73 \%$ of the reported lesions to be located occlusally [16]. Assuming eight occlusal molar surfaces being present and deducing the reported number of restorations (again assuming $73 \%$ being located occlusally) resulted in a tooth-level prevalence of $45 \%$ for initial and advanced, and $22 \%$ for only advanced lesions, respectively. Note that the assumption of all occlusal lesions being located on molars is a simplification and might lead to some distortion. For Egypt, data on caries experience from Cairo, the metropolis region Giza belongs to, were available [17], with $65 \%$ of reported initial and advanced lesions being situated on occlusal surfaces. Again, we assumed the lesions to be distributed among eight occlusal surfaces, which resulted in a tooth-level prevalence of $12.9 \%$ for all (initial and advanced) and $8.1 \%$ for only advanced lesions, respectively.

\section{Statistical analysis}

Willis and Hyde [10, 11] have previously demonstrated that if the test positive rate is known this constrains the region of values that the sensitivity and specificity take in receiver operating characteristic (ROC) space. This region is constrained further if the prevalence is also known. Thus, an applicable region for the test sensitivity and specificity may be derived based on the test positive rate and prevalence. In essence, it represents the feasible set of (sensitivity, specificity) pairs for the test given the information we have collected on the test positive rate and prevalence. Furthermore since the test positive rate 
and prevalence vary with the test (visual or radiography), target disorder (all lesions or only advanced lesions) and setting (Germany and Egypt) the applicable region is particular to the respective combination of these.

After deriving the applicable region, each study is compared with this region by first deriving the maximum likelihood estimate (MLE) for the sensitivity and specificity subject to it being constrained to lie in the applicable region $[10,11]$. This is then compared with the observed sensitivity and specificity and low probability studies $(p<0.025)$ are excluded. The resulting tailored set of studies is then aggregated using a bivariate random effects model to derive estimates for the sensitivity and specificity [18] - this is the TMA estimate.

The test positive rate ranges used to derive the applicable region in the TMA corresponded to the $99 \%$ confidence intervals derived from the routine data collected from each of the settings. The ranges used for the prevalence rates were based on a plausible interval that contained the point prevalence estimates derived for the setting (Table 1).

There is no reliable statistical method for estimating the level of heterogeneity when using bivariate measures (sensitivity, specificity). However, the ROC plot provides a visual representation of the dispersion of studies which is indicative of the level of heterogeneity.

\section{Results}

From the original reviews [3, 6], 210 studies on visual caries detection and 100 studies on radiographic caries detection were included in our meta-analyses (see Additional file 1). Inspection of the ROC plots for the conventional meta-analyses demonstrates widespread dispersion of studies, suggesting that heterogeneity is present in all the analyses as expected. Figures 1 and 2 illustrate this for the radiographic detection of occlusal lesions and the visual detection of advanced occlusal lesions.
From the routine data interval estimates for the test positive rate and prevalence were calculated (Table 1) and these were used to derive an applicable region $[10,11]$ for each of the tests in Egypt and Germany. This is illustrated for the radiographic detection of occlusal lesions and the visual detection of advanced occlusal lesions in Figs. 1 and 2.

In Fig. 1 the applicable regions for Egypt and Germany are close and overlap in large areas of the ROC space, whereas in Fig. 2 they occupy discrete regions. Thus, the visual detection of advance occlusal lesions in Egypt is likely to have sensitivity and specificity which is in a completely different region of ROC space from that of Germany. Furthermore, from Fig. 2 the conventional point estimate (all studies included) lies between the applicable regions for both Egypt and Germany and is significantly different from the tailored estimate for Germany.

The location of the applicable region in ROC space affects the number of studies included in the tailored meta-analyses and in general, tailoring to the setting reduces the number of studies applicable (Table 2). Thus for the visual detection of all occlusal lesions out of the 67 studies included in the original meta-analysis only 51 studies were applicable to Egypt and only 12 studies were applicable to Germany. This can have profound effects on the estimates for the sensitivity and specificity which are plausible for these two settings.

Table 3 illustrates the effects tailoring has on the likelihood ratios for the test. The most marked differences occur with the visual detection of occlusal lesions. In contrast, radiographic detection is broadly consistent across both settings (Egypt and Germany) and in line with the average estimates when all studies are included.

The positive and negative predictive values (PPV, NPV) for the tests are given in Tables 4 and 5 using the point prevalence estimates for the settings where available. Again the differences between Egypt and Germany are large for visual detection and are driven by the differences in the prevalence and likelihood ratios. A PPV

Table 1 Test positive rate and prevalence rates used in tailored meta-analysis

\begin{tabular}{|c|c|c|c|c|c|c|c|c|}
\hline & \multicolumn{4}{|l|}{ Egypt } & \multicolumn{4}{|l|}{ Germany } \\
\hline & \multicolumn{2}{|c|}{ Test positive rate } & \multicolumn{2}{|l|}{ Prevalence } & \multicolumn{2}{|c|}{ Test positive rate } & \multicolumn{2}{|l|}{ Prevalence } \\
\hline & Lower limit & Upper limit & Lower limit & Upper limit & Lower limit & Upper limit & Lower limit & Upper limit \\
\hline \multicolumn{9}{|l|}{ Visual detection } \\
\hline advanced occlusal & 0.20 & 0.33 & 0.01 & 0.15 & 0.05 & 0.26 & 0.15 & 0.30 \\
\hline all occlusal & 0.30 & 0.44 & 0.05 & 0.20 & 0.13 & 0.28 & 0.35 & 0.55 \\
\hline \multicolumn{9}{|l|}{ Radiographic detection } \\
\hline advanced occlusal & 0.14 & 0.26 & 0.09 & 0.29 & 0.06 & 0.18 & 0.01 & 0.21 \\
\hline all occlusal & 0.14 & 0.26 & 0.09 & 0.29 & 0.06 & 0.19 & 0.01 & 0.21 \\
\hline advanced proximal & 0.02 & 0.07 & & & 0.03 & 0.08 & & \\
\hline all proximal & 0.03 & 0.08 & & & 0.07 & 0.14 & & \\
\hline
\end{tabular}

The lower and upper limits for the test positive rates are $99 \%$ confidence interval limits derived from the routine data. The lower and upper limits for the prevalence rates are plausible limits based on a priori point estimates 


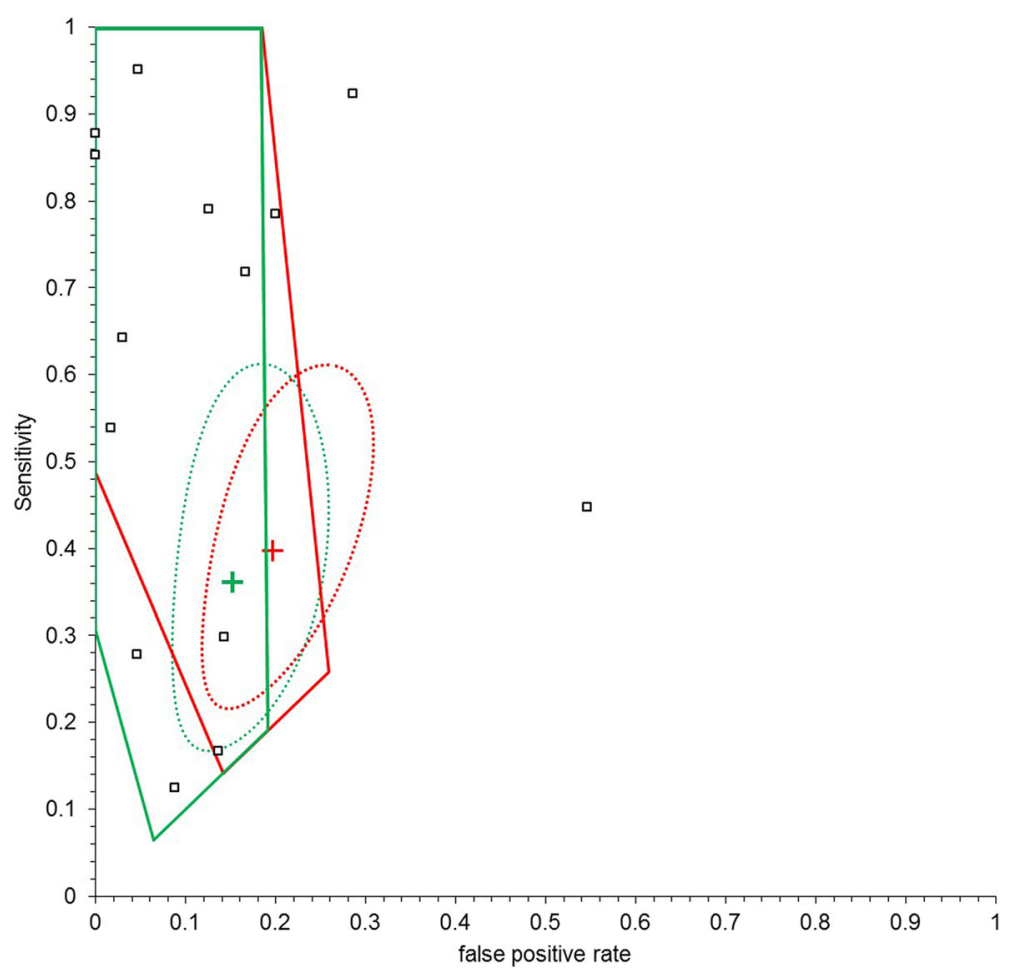

Fig. 1 Comparison of tailored meta-analyses for the radiographic detection of all occlusal lesions for Egypt (red) and Germany (green). Also given are the summary estimates (cross) with the associated 95\% confidence ellipses

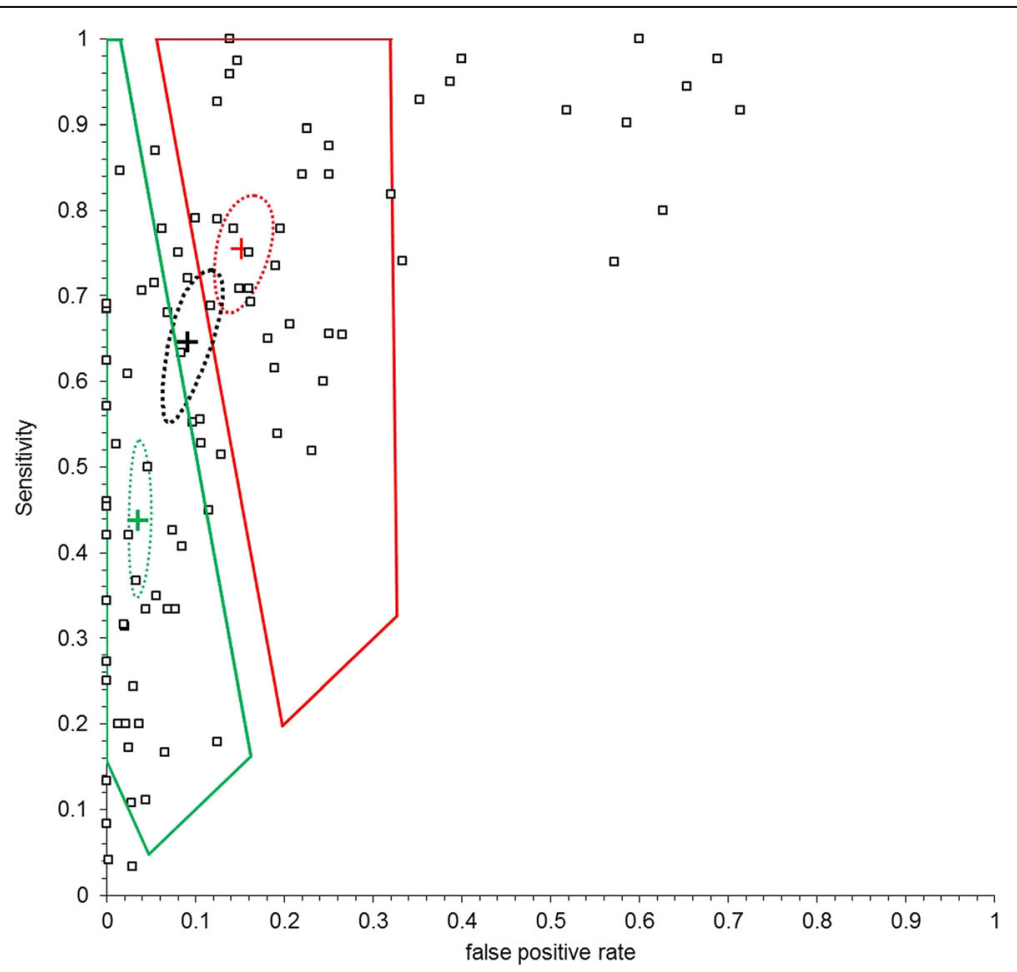

Fig. 2 Comparison of tailored meta-analyses for the visual detection of advanced occlusal lesions for Egypt (red), Germany (green) and the conventional meta-analysis (black). Also given are the summary estimates (cross) with the associated 95\% confidence ellipses 
Table 2 Mean $(95 \%$ Cl) sensitivity and specificity for all studies, tailored to Egypt and tailored to Germany

\begin{tabular}{|c|c|c|c|c|c|c|c|c|c|}
\hline & \multicolumn{3}{|c|}{ ALL } & \multicolumn{3}{|c|}{ Egypt } & \multicolumn{3}{|c|}{ Germany } \\
\hline & $n$ & Sensitivity & Specificity & $n$ & Sensitivity & Specificity & $\mathrm{n}$ & Sensitivity & Specificity \\
\hline \multicolumn{10}{|l|}{ Visual detection } \\
\hline advanced occlusal & 94 & $64.6 \%(57-71)$ & $90.9 \%(88-93)$ & 55 & $75.1 \%(70-81)$ & $84.9 \%(82-89)$ & 50 & $43.7 \%(37-51)$ & $96.5 \%(95-97)$ \\
\hline all occlusal & 67 & $85.5 \%(81-89)$ & $75.3 \%(70-80)$ & 51 & $85.8 \%(82-89)$ & $77.0 \%(73-81)$ & 12 & $49.1 \%(40-58)$ & $91.2 \%(88-94)$ \\
\hline \multicolumn{10}{|l|}{ Radiographic detection } \\
\hline advanced occlusal & 44 & $52.3 \%(44-60)$ & $89.3 \%(86-92)$ & 38 & $54.2 \%(47-61)$ & 89.1\% (86-92) & 36 & $50.8 \%(43-59)$ & $91.4 \%(88-94)$ \\
\hline all occlusal & 14 & $38.6 \%(25-54)$ & $77.8 \%(70-84)$ & 11 & $39.7 \%(25-56)$ & $80.4 \%(72-87)$ & 9 & $36.0 \%(20-56)$ & $84.8 \%(77-90)$ \\
\hline advanced proximal & 22 & $44.4 \%(38-51)$ & $95.5 \%(94-97)$ & 22 & $44.4 \%(38-51)$ & $95.5 \%(94-97)$ & 22 & $44.4 \%(38-51)$ & $95.5 \%(94-97)$ \\
\hline all proximal & 38 & $43.2 \%(36-51)$ & $90.0 \%(87-92)$ & 31 & $40.2 \%(32-48)$ & $92.5 \%(91-94)$ & 34 & $40.5 \%(33-48)$ & $91.7 \%(90-93)$ \\
\hline
\end{tabular}

of $82 \%$ for visual detection of all occlusal lesions in the German setting suggests it should be used for ruling in occlusal lesions when the test is positive, whilst in Egypt the same test performs with a PPV of $36 \%$. In contrast, a negative test result is more useful to clinicians in Egypt $(\mathrm{NPV}=97 \%)$ compared with an NPV of $69 \%$ in the German setting.

\section{Discussion}

When identifying carious lesions, general dental practitioners are faced with a specific patient population, where the prevalence rates of different lesions (and stages) are not always represented by those obtained from individual studies. Moreover, each practitioner will apply methods differently, yielding different test positive rates in the hands of different dentists. As a result, the PPV and/or NPV from a DAS are possibly very different from those found in a specific practice. The resulting over- and under-detection have implications both on a clinical and a public health level $[4,5]$.

When deciding if and how to apply a caries detection method, dentists should be cautious not to solely rely on the aggregate estimates from conventional diagnostic test meta-analyses particularly if there is widespread heterogeneity, as the implicit assumption of 'one-size fits all' may be untenable. The heterogeneity [19] stemming from factors like study design, test execution, threshold and patient spectrum can be generally described. However, it is difficult to determine which factors are responsible for the result of heterogeneity between DAS, and these cannot be addressed by conventional meta-analysis. Tailored meta-analysis circumvents this by using information directly from the setting of interest to determine which sub-group of studies are likely to reflect the performance in that setting.

We used tailored meta-analysis to assess whether the summary estimate of all DAS included in conventional meta-analysis is accurate in a setting such as Germany or Egypt. Including setting-specific test positive rates and prevalence allowed us to tailor our diagnostic meta-analysis. We showed that for visual detection of occlusal lesions the tailored meta-analysis estimates for Egypt and Germany do not coincide whereas for radiographic detection they do. This may have a number of reasons. First, test conduct may differ, starting from who performed the examinations (both visual and radiograpically) and how exactly this was done. Obviously, these aspects will not be $100 \%$ standardized even under controlled settings, and it is impossible to standardize them across settings in routine care. Tailoring meta-analysis

Table 3 Mean (95\% CI) Positive and Negative Likelihood Ratios (LR) for all studies, tailored to Egypt and tailored to Germany

\begin{tabular}{|c|c|c|c|c|c|c|c|c|c|}
\hline & \multicolumn{3}{|c|}{ ALL } & \multicolumn{3}{|c|}{ Egypt } & \multicolumn{3}{|c|}{ Germany } \\
\hline & $n$ & Positive LR & Negative LR & $n$ & Positive LR & Negative LR & $n$ & Positive LR & Negative LR \\
\hline \multicolumn{10}{|l|}{ Visual detection } \\
\hline advanced occlusal & 94 & $7.09(5.6-8.9)$ & $0.39(0.3-0.5)$ & 55 & $5.0(4.3-5.8)$ & $0.29(0.2-0.4)$ & 50 & $12.5(9.2-16.9)$ & $0.58(0.5-0.7)$ \\
\hline all occlusal & 67 & $3.46(2.8-4.3)$ & $0.19(0.15-0.25)$ & 51 & $3.73(3.1-4.4)$ & $0.18(0.14-0.24)$ & 12 & $5.56(3.7-8.5)$ & $0.56(0.46-0.68)$ \\
\hline \multicolumn{10}{|l|}{ Radiographic detection } \\
\hline advanced occlusal & 44 & $4.90(3.7-6.5)$ & $0.53(0.46-0.62)$ & 38 & $5.96(3.7-6.6)$ & $0.51(0.44-0.60)$ & 36 & $5.87(4.3-8.0)$ & $0.54(0.46-0.63)$ \\
\hline all occlusal & 14 & $1.74(1.3-2.4)$ & $0.79(0.65-0.96)$ & 11 & $2.02(1.4-2.9)$ & $0.75(0.60-0.94)$ & 9 & $2.36(1.4-4.1)$ & $0.76(0.58-0.99)$ \\
\hline advanced proximal & 22 & $9.82(7.3-13.3)$ & $0.58(0.52-0.65)$ & 22 & $9.82(7.3-13.3)$ & $0.58(0.52-0.65)$ & 22 & $9.82(7.3-13.3)$ & $0.58(0.52-0.65)$ \\
\hline all proximal & 38 & $4.30(3.4-5.5)$ & $0.63(0.56-0.72)$ & 31 & $5.34(4.2-6.8)$ & $0.65(0.57-0.74)$ & 34 & $4.89(3.9-6.2)$ & $0.65(0.57-0.73)$ \\
\hline
\end{tabular}


Table 4 Positive Predictive Values (PPV) of different tests for Egypt and Germany where prevalence rates are available

\begin{tabular}{|c|c|c|c|c|c|c|}
\hline & \multicolumn{3}{|l|}{ Egypt } & \multicolumn{3}{|l|}{ Germany } \\
\hline & Prevalence & Positive LR & PPV & Prevalence & Positive LR & PPV \\
\hline \multicolumn{7}{|l|}{ Visual detection } \\
\hline advanced occlusal & $8.1 \%$ & 5.00 & $30.6 \%$ & $22.0 \%$ & 12.5 & $77.9 \%$ \\
\hline all occlusal & $12.9 \%$ & 3.73 & $35.6 \%$ & $45.0 \%$ & 5.56 & $82.0 \%$ \\
\hline \multicolumn{7}{|l|}{ Radiographic detection } \\
\hline advanced occlusal & $19.0 \%$ & 5.96 & $58.3 \%$ & $10.0 \%$ & 5.87 & $39.5 \%$ \\
\hline all occlusal & $19.0 \%$ & 2.02 & $32.1 \%$ & $11.0 \%$ & 2.36 & $22.6 \%$ \\
\hline
\end{tabular}

can be useful here to yield setting-specific estimates which are more applicable under the specific circumstances. Second, prevalence rates differed, as described, reflecting different health conditions and risk factors (diet, availability of fluoride, oral hygiene), but also test positive rates. For example, we only evaluated accessible surfaces, which usually meant for occlusal surfaces, unsealed ones. In Germany, unsealed surfaces are found mainly in patients with irregular utilization of dental services; these patients usually also show high caries risk [12]. Low risk patients usually attend the dentist regularly; the majority of occlusal surfaces in these surfaces are sealed for preventive reasons [12]. The resulting high test positive rate for caries lesions in the available surfaces is a result of this; consequently, visual detection had high specificity and PPV, but relatively low NPV. Hence, on occlusal surfaces, dentists in this specific German setting can expect positive detections of caries on occlusal surfaces to be true, and treat accordingly. Negative detections, in contrast, may be false; an additional (more sensitive) diagnostic measure could be applied additionally to increase the NPV. In contrast, in Egypt the tooth level prevalence of occlusal lesions was very low, resulting in low specificity and low PPV of visual detection. Hence, positive detections should be regarded with caution and an additional test for verifying the positive test result should be considered prior to applying (invasive) treatments.

In contrast, for radiographic assessments, tailored meta-analysis did not yield significantly different findings for both settings or compared to conventional meta-analysis. This may have a number of reasons, too. First, we did not tailor for prevalence of proximal lesions; this was, as no epidemiologic data for both settings was available. Hence, our estimates for radiographic detection are not as tailored as those for visual detection. Second, prevalence rates may indeed be similar in both settings (something we don't know) for proximal lesions. Third, it can be assumed that interpreting radiographs is -to some degree- more objective than visual assessment; the impact of test conduct may be lower for radiographic than for visual detection. Fourth, heterogeneity was generally lower for DSA on radiographic detection; the impact of tailoring will automatically be lower under such circumstances. Last, the number of studies included in this meta-analysis was lower; confidence intervals for any accuracy estimates were wider due to lower statistical power and differences between settings less likely to be detected.

\section{Limitations}

The main driver of tailored meta-analysis is the interval estimate for the test positive rate which determines the size of the applicable region. The interval estimate for the prevalence helps refine the applicable region further. Thus the estimates may be subject to selection bias. This was mitigated by sampling consecutive patients although it is still possible that an unrepresentative sample was selected.

Table 5 Negative Predictive Values (NPV) of different tests for Egypt and Germany where prevalence rates are available

\begin{tabular}{|c|c|c|c|c|c|c|}
\hline & \multicolumn{3}{|l|}{ Egypt } & \multicolumn{3}{|l|}{ Germany } \\
\hline & Prevalence & Negative LR & NPV & Prevalence & Negative LR & NPV \\
\hline \multicolumn{7}{|l|}{ Visual detection } \\
\hline advanced occlusal & $8.1 \%$ & 0.29 & $97.5 \%$ & $22.0 \%$ & 0.58 & $85.9 \%$ \\
\hline all occlusal & $12.9 \%$ & 0.18 & $97.4 \%$ & $45.0 \%$ & 0.56 & $68.6 \%$ \\
\hline Radiographic detection & $19.0 \%$ & 0.51 & $89.3 \%$ & $10.0 \%$ & 0.54 & $94.3 \%$ \\
\hline advanced occlusal & $19.0 \%$ & 0.75 & $85.0 \%$ & $11.0 \%$ & 0.76 & $91.4 \%$ \\
\hline all occlusal & $8.1 \%$ & 0.29 & $97.5 \%$ & $22.0 \%$ & 0.58 & $85.9 \%$ \\
\hline
\end{tabular}


Only certain surfaces on molars were assessed as both visual occlusal and radiographic proximal assessment are useful detection methods here. In practice, dentists would assess other teeth and surfaces, too. This may affect the prevalence rates reported in this study.

Many of the primary studies were conducted in vitro and showed high risk of bias, often due to unrealistically high prevalence of lesions and potential spectrum bias. However, when there are multiple sources of heterogeneity the individual contributions of the different sources of heterogeneity are unlikely to be determined. In such an instance, tailored meta-analysis may afford the advantage of being probabilistic rather than deterministic in its study selection.

Some surfaces were not accessible for inspection due to teeth not being fully erupted, teeth overlapping on radiographs, presence of sealants or presence of restorations. The presence of a restoration indicates a previous lesion on the surface and omitting such cases from the data could potentially lead to the test positive rate being underestimated. However, there were only a few such cases and their effect on our estimates is likely to be limited.

\section{Recommendations}

Diagnostic accuracy studies should be scrutinized for their applicability to different settings, and a critical risk of bias assessment with a focus on setting-specific prevalence and patient spectrum should be performed. A systematic assessment of the risk of bias may be made using tools such as QUADAS-2 [20] prior to the study conduct to allow a higher internal validity.

It is clear from this analysis and others $[10,11]$ that the summary estimates yielded from conventional meta-analysis of diagnostic accuracy studies have the potential to be inapplicable in particular clinical settings and tailored meta-analysis potentially overcomes this. Although at presence it remains the reserve of statisticians, a user-friendly package in which medical practitioners could readily implement it in the clinical setting would widen the access of the technique.

One of the difficulties for systematic reviewers is the inadequate reporting of data in the primary studies. The STARD statement, which provides standards on reporting of primary diagnostic studies, aimed to address this [21]. Although any improvements in reporting remain largely the responsibility of primary research investigators, journals have a role in facilitating this process and ensuring the STARD statement is fully observed in published diagnostic test accuracy studies.

Although tailored meta-analysis produces estimates which are plausible, their validity is yet to be established. Recently there have been developments in the evaluation of the validity of meta-analysis estimates [22]. Potentially such methods could be used to investigate the validity of tailored meta-analysis findings, but at present this an area for future research.

Existing or newly developed guidelines on caries detection methods should include not only generic, but tailored accuracy estimates, where possible, before deciding to recommend or refute the application of a detection method. Routinely collected data (which is increasingly available) might be useful for such tailoring. In the long term, tailoring might be performed using routine data via practice software, with generated outputs assisting practitioners in individualized decision-making.

\section{Conclusions}

Conventional test accuracy meta-analyses may yield aggregate estimates which are inapplicable to specific settings due to the failure of the summary estimate to capture the variation in test performance across different settings. However, routine data collected from the setting of interest may be combined with secondary research to modify this estimate to produce an estimate which is tailored to the setting. This estimate is more likely to be representative of the test accuracy within the setting of interest. Test accuracy meta-analyses should be scrutinized for their applicability to different settings and tailoring may eventually be useful for individualized decision-making.

\section{Additional file}

Additional file 1: All data used the in the meta-analysis, tabulated. (ZIP $105 \mathrm{~kb})$

\section{Abbreviations}

DAS: Diagnostic accuracy study; ICDAS: International Caries Detection and Assessment System; MLE: Maximum likelihood estimate; NPV: Negative predictive value; PPV: Positive predictive value; ROC: Receiver Operating Characteristics; TMA: Tailored Meta-Analysis

\section{Funding}

BHW is in receipt of an Medical Research Council Clinician Scientist's award (MR/N007999/1). The funding body had no role in the design of the study and collection, analysis, and interpretation of data and in writing the manuscript.

\section{Availability of data and materials}

All data on the meta-analysis is available in this manuscript and the Additional file 1 . The raw unaggregated, anonymized routine data is available from the authors on request.

\section{Authors' contributions}

FS and BW developed the study, analyzed and interpreted the data. FS collected data and wrote the manuscript. KE, OES, RM collected data, interpreted the findings and provided feedback to the manuscript. TG and FMM provided data, interpreted the findings and provided feedback to the manuscript. All authors read and approved the final manuscript.

\section{Ethics approval and consent to participate}

All procedures performed in studies involving human participants were in accordance with the ethical standards of the institutional and/or national research committee and with the 1964 Helsinki declaration and its later amendments or comparable ethical standards. The routine data use was granted by the Charité ethics (EA2 137/14) and the Cairo university ethics $(16 / 7 / 3)$ 


\section{Consent for publication}

The routine data use was granted by the Charite ethics (EA2 137/14) and the Cairo university ethics (16/7/3). No separate informed consent procedure for publication was needed, mainly only aggregate data are published.

\section{Competing interests}

The authors declare that they have no competing interests.

\section{Publisher's Note}

Springer Nature remains neutral with regard to jurisdictional claims in published maps and institutional affiliations.

\section{Author details}

'Department of Operative and Preventive Dentistry, Charité Universitätsmedizin Berlin, Aßmannshauser Str. 4-6, 14197 Berlin, Germany. ${ }^{2}$ Department of Pediatric Dentistry, Cairo University, Giza, Egypt. ${ }^{3}$ Department of Pediatric Dentistry, Cairo University, 11 el Saraya Street, Manial, Cairo, Egypt. ${ }^{4}$ Department of Pediatric Dentistry, School of Dentistry, University of São Paulo, Av. Lineu Prestes, São Paulo 2227, Brazil. ${ }^{5}$ Institute of Applied Health Research, University of Birmingham, Edgbaston, Birmingham B15 2TT, UK.

Received: 25 July 2017 Accepted: 22 May 2018

Published online: 08 June 2018

\section{References}

1. Baelum V, Heidmann J, Nyvad B. Dental caries paradigms in diagnosis and diagnostic research. Eur J Oral Sci. 2006;114(4):263-77.

2. Baelum V, Hintze H, Wenzel A, Danielsen B, Nyvad B. Implications of caries diagnostic strategies for clinical management decisions. Community Dent Oral Epidemiol. 2012:40(3):257-66.

3. Gimenez T, Piovesan C, Braga MM, Raggio DP, Deery C, Ricketts DN, Ekstrand KR, Mendes FM. Visual inspection for caries detection: a systematic review and meta-analysis. J Dent Res. 2015;94(7):895-904

4. Schwendicke F, Paris S, Stolpe M. Detection and treatment of proxima caries lesions: milieu-specific cost-effectiveness analysis. J Dent. 2015; 43(6):647-55.

5. Schwendicke F, Stolpe M, Meyer-Lueckel H, Paris S. Detecting and treating occlusal caries lesions: a cost-effectiveness analysis. J Dent Res. 2015;94(2):272-80

6. Schwendicke F, Tzschoppe M, Paris S. Radiographic caries detection: a systematic review and meta-analysis. J Dent. 2015;43(8):924-33.

7. Willis $\mathrm{BH}$. Spectrum bias-why clinicians need to be cautious when applying diagnostic test studies. Fam Pract. 2008:25(5):390-6.

8. Willis BH, Quigley M. Uptake of newer methodological developments and the deployment of meta-analysis in diagnostic test research: a systematic review. BMC Med Res Methodol. 2011;11(1):1-8.

9. Mendes FM, Novaes TF, Matos R, Bittar DG, Piovesan C, Gimenez T, Imparato JC, Raggio DP, Braga MM. Radiographic and laser fluorescence methods have no benefits for detecting caries in primary teeth. Caries Res. 2012;46(6):536-43.

10. Willis BH, Hyde CJ. Estimating a test's accuracy using tailored metaanalysis - how setting-specific data may aid study selection. J Clin Epidemiol. 2014;67(5):538-46

11. Willis BH, Hyde CJ. What is the test's accuracy in my practice population? Tailored meta-analysis provides a plausible estimate. J Clin Epidemiol. 2015;68(8):847-54.

12. Micheelis W, Schiffner U. Vierte Deutsche Mundgesundheits-Studie (DMS IV). In: IDZ Materialreihe. Edited by Institut der Deutschen Zahnärzte, vol. 31. Deutscher Ärzteverlag, Cologne; 2006.

13. DAJ. Epidemiologische Begleituntersuchungen zur Gruppenprophylaxe 2000. In: Gutachten aus den Bundesländern bzw Landesteilen Deutsche Arbeitsgemeinschaft für Jugendzahnpflege; 2001.

14. Ismail Al, Sohn W, Tellez M, Amaya A, Sen A, Hasson H, Pitts NB. The international caries detection and assessment system (ICDAS): an integrated system for measuring dental caries. Community Dent Oral Epidemiol. 2007; 35(3):170-8

15. Pieper K, Weber K, Margraf-Stiksrud J, Heinzel-Gutenbrunner M, Stein S, Jablonski-Momeni A. Evaluation of a preventive program aiming at children with increased caries risk using ICDAS II criteria. Clin Oral Investig. 2013; 17(9):2049-55.
16. Jablonski-Momeni A, Winter J, Petrakakis P, Schmidt-Schäfer S. Caries prevalence (ICDAS) in 12-year-olds from low caries prevalence areas and association with independent variables. Int J Paediatr Dent. 2013;24(2):90-7.

17. Mobarak EH, Shabayek MM, Mulder J, Reda AH, Frencken JE. Caries experience of Egyptian adolescents: does the atraumatic restorative treatment approach offer a solution? Med Princ Pract. 2011;20(6):545-9.

18. Chu H, Cole SR. Bivariate meta-analysis of sensitivity and specificity with sparse data: a generalized linear mixed model approach. J Clin Epidemiol. 2006;59(12):1331-2. author reply 1332-1333

19. Whiting P, Rutjes AW, Reitsma JB, Glas AS, Bossuyt PM, Kleijnen J. Sources of variation and bias in studies of diagnostic accuracy: a systematic review. Ann Intern Med. 2004;140(3):189-202.

20. Whiting PF, Rutjes AW, Westwood ME, Mallett S, Deeks JJ, Reitsma JB, Leeflang MM, Sterne JA, Bossuyt PM, QUADAS-2 Group. QUADAS-2: a revised tool for the quality assessment of diagnostic accuracy studies. Ann Intern Med. 2011;155(8):529-36.

21. Bossuyt PM, Reitsma JB, Bruns DE, Gatsonis CA, Glasziou PP, Irwig LM, Moher D, Rennie D, de Vet HC, Lijmer JG, Standards for Reporting of Diagnostic Accuracy. The STARD statement for reporting studies of diagnostic accuracy: explanation and elaboration. Ann Intern Med. 2003; 138(1):W1-12.

22. Willis $\mathrm{BH}$, Riley RD. Measuring the statistical validity of summary metaanalysis and meta-regression results for use in clinical practice. Stat Med. 2017:36(21):3283-301.

\section{Ready to submit your research? Choose BMC and benefit from:}

- fast, convenient online submission

- thorough peer review by experienced researchers in your field

- rapid publication on acceptance

- support for research data, including large and complex data types

- gold Open Access which fosters wider collaboration and increased citations

- maximum visibility for your research: over $100 \mathrm{M}$ website views per year

At BMC, research is always in progress.

Learn more biomedcentral.com/submissions 